\title{
CONDITIONS FOR RESTRICTED TRANSLATION OPERATORS TO BELONG TO $S_{p}$
}

\author{
HARRY DYM AND ISIDOR SHAPIRO
}

\begin{abstract}
Let $B$ be a Blaschke product, let $P$ be the orthogonal projection of $L^{2}\left(R^{1}\right)$ onto the left invariant subspace $H^{2} \Theta B H^{2}$ and let $E_{t}: f(\gamma) \rightarrow$ $e^{-i \gamma t} f(\gamma)$. Conditions are given on the roots of $B$ for $P E_{t} P$ to belong to $S_{p}$, $0<p<\infty$.
\end{abstract}

1. Introduction. This paper gives conditions for membership in $S_{p}, 0<p<$ $\infty$, for the operators $K_{t}=P E_{t} P, t>0$, in which $E_{t}$ designates multiplication by $e^{-i \gamma t}: E_{t} f(\gamma)=e^{-i \gamma t} f(\gamma)$, and $P$ stands for the orthogonal projection of $L^{2}\left(R^{1}\right)$ onto the "left invariant" subspace $H^{2} \ominus B H^{2}$. Here $H^{2}$ is the Hardy space of class 2 over the upper-half plane, and

$$
B(\gamma)=\prod_{k=1}^{\infty}\left(\frac{1-\gamma / \omega_{k}}{1-\gamma / \omega_{k}^{*}}\right)
$$

is the Blaschke product with infinitely many roots [poles]

$$
\omega_{k}=a_{k}+i b_{k} \quad\left[\omega_{k}^{*}=a_{k}-i b_{k}\right], \quad b_{k}>0,
$$

which are subject to the usual constraint

$$
\sum_{k=1}^{\infty} \frac{b_{k}}{\left|\omega_{k}\right|^{2}+1}<\infty
$$

Recall that $K_{t}$ belongs to $S_{p}, 0<p \leqslant \infty$, if (1) it is compact, and (2) its $s$ numbers belong to $l_{p}$. The $s$ numbers, $s_{1}(t), s_{2}(t), \ldots$, of $K_{t}$ are the positive square roots of the eigenvalues of $K_{t}^{*} K_{t}$ arranged in decreasing size: $s_{1}(t) \geqslant$ $s_{2}(t) \geqslant \ldots$ Gohberg and Krein [2] is recommended for additional information on these spaces.

Let

$$
\alpha=\alpha_{B}=\limsup _{|a| \rightarrow \infty} \sum_{k=1}^{\infty} \frac{2 b_{k}}{\left|\omega_{k}-a\right|^{2}}, \quad a \in R^{1}
$$

and let

Received by the editors June 6, 1976 and, in revised form, October 20, 1976.

AMS (MOS) subject classifications (1970). Primary 47B10; Secondary 47A15, 47D05.

Key words and phrases. Compact operators, trace class, Hilbert Schmidt class, $S_{p}$ class, translation invariant subspaces, semigroups. 


$$
\beta=\beta_{B}=\text { the abscissa of convergence of } \sum_{n=1}^{\infty} e^{-b_{n} t} \text {. }
$$

AMPlification. If $b_{1} \leqslant b_{2} \leqslant \ldots$, then

$$
\beta=\limsup _{n \uparrow \infty} \frac{\log n}{b_{n}}
$$

see p. 45 of Widder [7].

The chief conclusions of this paper are:

TheOREM 2. In order for $K_{t}$ to belong to $S_{p}, 0<p<\infty$, it is sufficient that $t>[\sqrt{(\beta / p)}+\sqrt{\alpha}]^{2}$, and it is necessary that $t \geqslant \max [(\beta / p), \alpha]$.

THEOREM 3. $K_{t}$ belongs to $S_{p}, 0<p<\infty$, for every $t>0$ if and only if $\alpha=\beta=0$.

Theorem 3 is an immediate corollary of Theorem 2 . The condition $\beta=0$ means that $b_{n}$ must tend to $\infty$ rapidly enough as $n \uparrow \infty$ so that $\sum_{n=1}^{\infty} e^{-b_{n} t}<\infty$ for every $t>0$. It is interesting to compare the conclusions of Theorem 3 with those of Koosis [3] and Lax [4] who proved that $K_{t}$ is compact for every $t>0$ if and only if $\alpha=0$ and $\lim _{n \uparrow \infty} b_{n}=\infty$. The proof of Theorem 2 is given in §4. It depends in part upon the following refinement of the Koosis-Lax conditions for compactness.

THEOREM 1. In order for $K_{t}$ to be compact for a fixed $t>0$, it is necessary that $t \geqslant \alpha$ and $\lim _{n \uparrow \infty} b_{n}=\infty$, and it is sufficient that $t>\alpha$ and $\lim _{n \uparrow \infty} b_{n}=$ $\infty$.

This refinement must have been known to Moeller and Frederickson since the estimates used in their paper [6] can be adapted to prove Theorem 1. Nevertheless, since the result is not stated explicitly there, and the connections between their work and ours are not altogether transparent, a proof seems to be called for. The details will be given in $\S 3$.

Finally, it should be noted that the space $H^{2} \ominus B H^{2}$ is the image under the Fourier transform of the closed linear span in $L^{2}(0, \infty)$ of the functions

$$
e^{-i \omega_{k}^{*} x}, \quad k=1,2 \ldots
$$

augmented by

$$
x e^{-i \omega_{k}^{*} x}, x^{2} e^{-i \omega_{k}^{*} x}, \ldots, x^{m-1} e^{-i \omega_{k}^{*} x}
$$

in case that $\omega_{k}$ is a root of order $m$ of the Blaschke product $B$; see Lax [4] for help with this. In this formulation $K_{t}$ corresponds to left translation in the amount $t$, and the fact that $K_{t}$ is a semigroup of bounded operators: $K_{t+s}=$ $K_{t} K_{s}$ becomes transparent. The semigroup property implies that $K_{t+s}$ belongs to $S_{p}, 0<p \leqslant \infty$, for every $s \geqslant 0$ as soon as $K_{t}$ does. 
2. Preliminaries. In this section a number of facts and elementary estimates are prepared for future use.

Notation. $H^{1}, H^{2}, H^{\infty}$, denote the Hardy spaces of ciasses 1,2 and $\infty$, respectively, over the upper half-plane, $\omega^{*}=a-i b$ denotes the complex conjugate of $\omega=a+i b$, and the limits of integration in all unmarked integrals such as $\int f(\gamma) d \gamma$ are $\pm \infty$. The orthogonal projection of $L^{2}\left(R^{1}\right)$ onto $H^{2}\left[H^{2} \ominus B H^{2}\right]$ is designated by $\mathfrak{p}[P]$.

LEMMA 1. $K_{t}=\mathfrak{p} E_{t} P$ and $K_{t+s}=K_{t} K_{s}$ for $s, t \geqslant 0$.

Proof. An easy computation shows that $\mathfrak{p} E_{t} P$ maps $H^{2} \ominus B H^{2}$ into itself. Therefore $K_{t}=P E_{t} P=P \mathfrak{p} E_{t} P=\mathfrak{p} E_{t} P$. This justifies the first assertion. The second is self-evident from the remarks in the introduction. For a direct proof in the language of $K_{t}$ see Dym [1].

LEMMA 2. The set of functions

$$
\phi_{n}(\gamma)=\sqrt{\frac{b_{n}}{\pi}} \prod_{k=n}^{\infty} \frac{1-\gamma / \omega_{k}}{1-\gamma / \omega_{k}^{*}} /\left(\gamma-\omega_{n}\right), \quad n=1,2, \ldots
$$

is an orthonormal basis for $H^{2} \ominus B H^{2}$.

Proof. The orthonormality is an easy consequence of Cauchy's formula. The completeness proof is slightly more involved and rests upon the observation (which the reader may verify directly) that if $f \in H^{2} \ominus B H^{2}$, then $f=B g^{*}$ with $g \in H^{2} \ominus B H^{2}$ (see Dym [1] for details). Therefore,

$$
\begin{aligned}
\int \phi_{n}(\gamma)[f(\gamma)]^{*} d \gamma & =\sqrt{\frac{b_{n}}{\pi}} \int\left(\prod_{k=1}^{n-1} \frac{1-\gamma / \omega_{k}^{*}}{1-\gamma / \omega_{k}} /\left(\gamma-\omega_{n}\right)\right) g(\gamma) d \gamma \\
& =\sum_{k=1}^{n} c_{k} g\left(\omega_{k}\right) \quad\left(c_{k} \neq 0\right),
\end{aligned}
$$

assuming, for the sake of a simpler exposition, that the roots of $B$ are distinct; otherwise some derivatives will intervene. But this makes it plain that if $f$ is orthogonal to all the $\phi_{n}, n=1,2, \ldots$, then $g$ must vanish at all points $\omega_{n}$, $n=1,2, \ldots$ This exhibits $g$ as an element of both $B H^{2}$ and $H^{2} \ominus B H^{2}$. Hence $g=0$ and $f=B g^{*}=0$.

Lemma 3. Let $P_{n}$ denote the orthogonal projection onto the closed linear span of $\phi_{k}, k \geqslant n$, and let

$$
B_{n}(\gamma)=\prod_{k=n}^{\infty}\left(\frac{1-\gamma / \omega_{k}}{1-\gamma / \omega_{k}^{*}}\right)
$$

Then 


$$
\left\|K_{t} P_{n}\right\| \leqslant e^{-b t} / \inf _{a}\left|B_{n}(a+i b)\right|
$$

for any choice of $b<\inf \left\{b_{k}: k \geqslant n\right\}$.

Proof. This bound is essentially due to Koosis [3] and may be deduced from the formula

$$
\left\|K_{t} P_{n}\right\|=\sup \left|\int \frac{e^{i \gamma t}}{B_{n}(\gamma)} f(\gamma) d \gamma\right|, \quad f \in H^{1},\|f\|_{1}=1,
$$

for the norm of $K_{t} P_{n}=\mathfrak{p} E_{t} P P_{n}=\mathfrak{p} E_{t} P_{n}=P_{n} E_{t} P_{n}$ by displacing the line of integration into the upper half-plane. The formula for the norm is implicit in the work of Koosis [3]; an explicit proof may also be found in Dym [1]. The stated identification of $K_{t} P_{n}$ follows from Lemmas 1 and 2.

Lemma 4. The bounds

$$
\begin{array}{r}
\exp \left\{\frac{2 b v}{|\omega-a|^{2}}\left[1-\frac{\delta^{2}}{1-\delta^{2}}\right]\right\} \leqslant\left|\frac{1-i b /\left(\omega^{*}-a\right)}{1-i b /(\omega-a)}\right| \\
\leqslant \exp \left\{\frac{2 b v}{|\omega-a|^{2}}\left[1+\frac{\delta^{2}}{1-\delta^{2}}\right]\right\}
\end{array}
$$

prevail for every pair of points $a+i b$, and $\omega=u+i v$ in the open upper half-plane with $b / v \leqslant \delta<1$.

Proof. This is a refinement of an estimate due to Koosis [3] and is similar in content to Lemma 4 of [6]. The details can be carried out much as in the proof of Lemma 3 of Dym [1]. Indeed you have only to set $\xi=i b$ and $\zeta=\omega-a$ in that paper, and to check that the real part of $\alpha|\xi / \zeta|$ is bounded by $\delta^{2}\left(1-\delta^{2}\right)^{-1}$ since only the summands with odd index in the sum on the top of p. 396 in [1] intervene.

LeMmA 5. If $\lim _{k \uparrow \infty} b_{k}=\infty$, then for any $\varepsilon>0$ and any $0<\delta<1$, there exists an $n_{0}$ such that $\left\|K_{t} P_{n}\right\| \leqslant \exp \left\{-\delta b_{n}\left[t-(\alpha+\varepsilon)\left(1-\delta^{2}\right)^{-1}\right]\right\}$ for every $n>n_{0}$.

Proof. Given $\varepsilon$ and $\delta$ choose $n_{0}$ so large that $b \leqslant \delta b_{k}$ for $k \geqslant n_{0}$ and $\sum_{k=n}^{\infty} 2 b_{k} /\left|\omega_{k}-a\right|^{2} \leqslant \alpha+\varepsilon$ for $n \geqslant n_{0}$. Then, by Lemma 4 ,

$$
\begin{aligned}
\left|B_{n}(a+i b)\right|^{-1} & \leqslant \exp \left\{\sum_{k=n}^{\infty} \frac{2 b b_{k}}{\left|\omega_{k}-a\right|^{2}}\left(1-\delta^{2}\right)^{-1}\right\} \\
& \leqslant \exp \left\{b(\alpha+\varepsilon)\left(1-\delta^{2}\right)^{-1}\right\}
\end{aligned}
$$

for $n \geqslant n_{0}$. To finish, invoke Lemma 3 with $b=\delta b_{n}$. 
LEMMA 6. The eigenvalues $\lambda_{k}(t), k=1,2, \ldots$, of $K_{t}$ are in $1: 1$ correspondence with the roots $\omega_{k}, k=1,2, \ldots$, of $B$ :

$$
\lambda_{k}(t)=e^{-i \omega_{k}^{*} t}
$$

The corresponding system of root vectors is complete.

Proof. In the case of distinct roots it is easily checked that $K_{t} \psi_{k}=\lambda_{k} \psi_{k}$ with $\lambda_{k}=\lambda_{k}(t)=\exp \left(-i \omega_{k}^{*} t\right)$ and $\psi_{k}(\gamma)=\left(\gamma-\omega_{k}^{*}\right)^{-1}$.

If, say, $\omega_{1}$ has multiplicity $p$, i.e., $\omega_{1}=\omega_{2}=\cdots=\omega_{p}$, then the corresponding root vectors are given by

$$
\psi_{k}(\gamma)=\left(\gamma-\omega_{1}^{*}\right)^{-k}, \quad k=1, \ldots, p,
$$

and a routine calculation, based upon the fact that

$$
\begin{aligned}
\left(K_{t} \psi_{k}\right)(\omega) & =\int \frac{e^{-i \gamma t}}{\gamma-\omega}\left(\frac{1}{\gamma-\omega_{1}^{*}}\right)^{k} \frac{d \gamma}{2 \pi i} \\
& =-\left.\frac{1}{(k-1) !} \frac{d^{k-1}}{d \gamma^{k-1}}\left[\frac{e^{i \gamma t}}{\gamma-\omega}\right]\right|_{\gamma=\omega_{i}^{*}}
\end{aligned}
$$

for points $\omega$ in the open upper half-plane, leads to the identity

$$
K_{t} \psi_{k}=e^{-i \omega_{i}^{*} t}\left[\sum_{j=1}^{k} \frac{(-i t)^{k-j}}{(k-j) !} \psi_{j}\right]
$$

This exhibits $\psi_{1}, \ldots, \psi_{p}$ as root vectors associated with the eigenvalue $\exp \left(-i \omega_{1}^{*} t\right)$. The full set of root vectors is complete in $H^{2} \ominus B H^{2}$ because any $f$ in that space which is orthogonal to all of them belongs to both $B H^{2}$ and $H^{2} \ominus B H^{2}$ and so must vanish.

3. Conditions for compactness. The next two lemmas are due to Koosis [3]. For the sake of completeness and the convenience of the reader, new and somewhat quicker proofs will be given.

Notation. $(f, g)$ denotes the standard inner product $\int f g^{*}$ in $L^{2}$.

LEMMA 7. Let $P_{n}$ denote the orthogonal projection introduced in Lemma 3. Then $K_{t}$ is compact if and only if $\left\|K_{t} P_{n}\right\| \rightarrow 0$ as $n \uparrow \infty$.

Proof. If $\left\|K_{t} P_{n}\right\| \rightarrow 0$, as $n \uparrow \infty$, then $K_{t}=K_{t}\left(I-P_{n}\right)+K_{t} P_{n}$ can be approximated in norm by the finite dimensional operator

$$
K_{t}\left(I-P_{n}\right): f \rightarrow \sum_{k=1}^{n-1}\left(f, \phi_{k}\right) K_{t} \phi_{k},
$$

and so $K_{t}$ must be compact. Conversely, if $K_{t}$ is compact, then for every choice of $n, n=1,2, \ldots$, there exists an element $u_{n}$ in $P_{n}\left(H^{2} \ominus B H^{2}\right)$ with $\left\|u_{n}\right\|=1$ such that 


$$
\left\|K_{t} P_{n}\right\|=\left\|K_{t} P_{n} u_{n}\right\|=\left\|K_{t} u_{n}\right\|
$$

Moreover, the bound

$$
\left|\left(u_{n}, g\right)\right|^{2}=\left|\left(u_{n}, P_{n} g\right)\right|^{2} \leqslant\left\|P_{n} g\right\|^{2}=\sum_{k=n}^{\infty}\left|\left(g, \phi_{k}\right)\right|^{2},
$$

which is valid for every $g \in L^{2}$, implies that $u_{n}$ tends weakly to zero in $L^{2}$ as $n \uparrow \infty$. Hence,

$$
\lim _{n \uparrow \infty}\left\|K_{t} P_{n}\right\|=\lim _{n \uparrow \infty}\left\|K_{t} u_{n}\right\|=0
$$

since $K_{t}$ is compact, and the proof is complete.

LEMMA 8. If $K_{t}$ is compact then $\lim _{k \uparrow \infty} b_{k}=\infty$.

Proof. This is immediate from Lemma 6 since the eigenvalues of an infinite dimensional compact operator must tend to 0 .

Proof of Theorem 1. The sufficiency is immediate from Lemma 7 and the bound of Lemma 5: You have only to choose $\varepsilon$ and $\delta$ in the latter so as to be positive and yet small enough so that $0<(\alpha+\varepsilon)\left(1-\delta^{2}\right)^{-1}<t$. The proof of necessity is adapted from Moeller and Frederickson [6]. A basic ingredient is an observation credited to Lax [4], [5] (although he credits it to Müntz and Szász), which in our notation amounts to the identity

$$
\left|B_{n}(a+i b)\right|=\left\|\left(I-P_{n}\right) \varphi\right\|_{2}
$$

for $\varphi=\varphi(\gamma)=(b / \pi)^{1 / 2}\left(\gamma-\omega^{*}\right)^{-1}$ and $\omega=a+i b$ with $b>0$. Because of it,

$$
\begin{aligned}
e^{-b t} & =\left\|e^{-i \omega^{*} t} \varphi\right\|_{2}=\left\|\mathfrak{p} E_{t} \varphi\right\|_{2} \\
& =\left\|K_{t} P_{n} \varphi+\mathfrak{p} E_{t}\left(I-P_{n}\right) \varphi\right\|_{2} \\
& \leqslant\left\|K_{t} P_{n}\right\|+\left|B_{n}(a+i b)\right| \\
& \leqslant\left\|K_{t} P_{n}\right\|+\exp \left\{-\sum_{k=n}^{\infty} \frac{2 b_{k} b}{\left|\omega_{k}-a\right|^{2}}\left(1-\frac{\delta^{2}}{1-\delta^{2}}\right)\right\}
\end{aligned}
$$

presuming, as you may in view of Lemma 8 , that $\left|b / b_{k}\right| \leqslant \delta<\frac{1}{2}$ for $k \geqslant n$ and $n$ large enough. But this implies that

$$
e^{-b t} \leqslant\left\|K_{t} P_{n}\right\|+\exp \left\{-\alpha b\left(1-\delta^{2}\right)^{-1}\left(1-2 \delta^{2}\right)\right\}
$$

and so, because of Lemma 7 , you have only to let $n \uparrow \infty$, and $\delta \downarrow 0$, in that order, to complete the proof. 


\section{Proof of Theorem 2.}

Proof OF SUfFicIENCY. The assumptions imply, in particular, that $t>\alpha$ and that $\lim _{n \uparrow \infty} b_{n}=\infty$. Therefore $K_{t}$ is compact, by Theorem 1 , and you may assume that the roots are indexed so as to have $b_{1} \leqslant b_{2} \leqslant \ldots$ Now the minimax characterization of eigenvalues implies that the $n$th $s$ number of $K_{t}$ is subject to the bound $s_{n}(t) \leqslant\left\|K_{t} P_{n}\right\|$. Therefore, by Lemma 5 , you see that for any $\varepsilon>0$ and $0<\delta<1$ there exists an $n_{0}$ such that

$$
\left[s_{n}(t)\right]^{p} \leqslant \exp \left\{-p \delta b_{n}\left[t-(\alpha+\varepsilon)\left(1-\delta^{2}\right)^{-1}\right]\right\}
$$

for all $n \geqslant n_{0}$. Consequently, $\sum_{n=1}^{\infty}\left[s_{n}(t)\right]^{p}$ will converge for

$$
t>\limsup _{n \uparrow \infty} \frac{\log \left|\sum_{k=1}^{n} \exp \left\{p \delta b_{k}(\alpha+\varepsilon)\left(1-\delta^{2}\right)^{-1}\right\}\right|}{p b_{n} \delta}
$$

as follows from the formula for the abscissa of convergence for Dirichlet series; see p. 45 of Widder [7]. But now the right-hand side is

$$
\begin{aligned}
& \leqslant \limsup _{n \uparrow \infty} \frac{\left[\log n+p \delta b_{n}(\alpha+\varepsilon)\left(1-\delta^{2}\right)^{-1}\right]}{p b_{n} \delta} \\
& \leqslant \frac{\beta}{p \delta}+\frac{(\alpha+\varepsilon)}{1-\delta^{2}} \leqslant \frac{\beta}{p \delta}+\frac{\alpha+\varepsilon}{1-\delta} ;
\end{aligned}
$$

and so in order for $K_{t}$ to belong to $S_{p}$ it suffices to have

$$
t>\beta / p \delta+\alpha /(1-\delta)
$$

for some choice of $0<\delta<1$. The final condition is attained by choosing $\delta$ so as to minimize the right-hand side.

Proof OF NECESSITY. To begin with, $K_{t}$ must be compact and so, by Theorem 1, you must have $t \geqslant \alpha$. Moreover, by Lemma 6 and p. 41 of Gohberg and Krein [2], it is also necessary that

$$
\sum_{n=1}^{\infty} e^{-b_{n} t p}=\sum_{n=1}^{\infty}\left|\lambda_{n}(t)\right|^{p}<\infty
$$

and, hence, that $p t \geqslant \beta$. The proof is complete.

\section{REFERENCES}

1. H. Dym, An extremal problem in the theory of Hardy functions, Israel J. Math. 18 (1974), 391-399.

2. I. C. Gohberg and M. G. Krein, Introduction to the theory of linear nonselfadjoint operators in Hilbert space, "Nauka", Moscow, 1965; English transl., Transl. Math. Monographs, vol. 18, Amer. Math. Soc., Providence, R. I., 1969. MR 36 \# 3137; 39 \#7447.

3. P. Koosis, Interior compact spaces of functions on a half-line, Comm. Pure Appl. Math. 10 (1957), 583-615. MR 20 \#224. 
4. P. Lax, Remarks on the preceding paper, Comm. Pure Appl. Math. 10 (1957), 617-622. MR 20 \#225.

5.

6. J. W. Moeller and P. O. Frederickson, $A$ density theorem for lacunary Fourier series, Bull. Amer. Math. Soc. 72 (1966), 82-86. MR 35 \# 2071.

7. D. V. Widder, The Laplace transform, Princeton Univ. Press, Princeton, N. J. 1941. MR 3, 232.

Department of Mathematics, The Weizmann Institute of Science, Rehovot, Israel 